\title{
Double-dot charge qubit and transport via dissipative cotunneling
}

\author{
Mei-Rong Li and Karyn Le Hur \\ Département de Physique, Université de Sherbrooke, Sherbrooke, Québec, Canada J1K 2R1
}

(Dated: November 6, 2018)

\begin{abstract}
We investigate transport through an exotic "charge" qubit composed of two strongly capacitively coupled quantum dots (QDs), each being independently connected to a side gate which in general exhibits a fluctuating electrostatic field (i.e., Johnson-Nyquist noise). Two quantum phases are found: the "Kondo" phase where an orbital-Kondo entanglement emerges and a "local moment" phase in which the noise destroys the Kondo effect leaving the orbital spin unscreened and resulting in a clear suppression of the conductance. In the Kondo realm, the transfer of charge across the setting is accompanied by zero-point charge fluctuations in the two dissipative environments and then the I-V characteristics are governed by what we call "dissipative cotunneling".
\end{abstract}

PACS numbers: 73.23.-b, 72.15.Qm, 42.50.Lc

The analogy between the Coulomb blockade effect in quantum dots (QDs) and the Kondo effect in heavy fermion systems has been emphasized for a decade [1]. Recently, fascinating quantum phase transitions were discovered in certain heavy fermion materials [2], and a spinboson-fermion impurity model has been proposed to capture the quantum passage from local moment magnetism to strongly-correlated Kondo behavior [3]. We seek to explore the emergence of similar physics at the mesoscopic scale through a setup composed of a double-QD "charge" qubit immersed in a noisy electromagnetic environment. The central goal of this Letter is to focus on transport properties close to the quantum phase transition. We stress that a strong capacitive coupling between dots is essential for the fabrication of the double-QD charge qubit. Two capacitively coupled QDs have recently attracted much attention [4]. One of the main motivations certainly lies in the fact that having coupled qubits is required for the controlled-NOT operation, one of the universal gates in quantum computing [5]. They are already known to exhibit non-trivial degeneracy points where quantum fluctuations can lead to exotic emergent states with orbital Kondo physics [6]. Experimentally, coupled QDs can be built up, e.g., in semiconducting heterostructures [7] and in nanotubes [8].

Model of two strongly capacitively coupled QDs subject to quantum noises. - The schematic circuit diagram of the setup under consideration is shown in Fig. 1. Each QD couples to a side-gate and each source of gate voltage is placed in series with an impedance $Z_{1,2}(\omega)$ showing resistive behavior at low frequency $\omega$. First, we will neglect the reservoirs of electrons (left and right leads) and seek to analyze the Coulomb blockade effect. At the HartreeFock level, the charging energy takes the form [5]

$$
\begin{aligned}
E_{n_{1} n_{2}}= & E_{c 1}\left(n_{g 1}-n_{1}\right)^{2}+E_{c 2}\left(n_{g 2}-n_{2}\right)^{2} \\
& +E_{m}\left(n_{g 1}-n_{1}\right)\left(n_{g 2}-n_{2}\right) ;
\end{aligned}
$$

we have $E_{c 1,2}=e^{2} C_{\Sigma 2,1} / 2\left(C_{\Sigma 1} C_{\Sigma 2}-C_{m}^{2}\right), E_{m}=$ $e^{2} C_{m} /\left(C_{\Sigma 1} C_{\Sigma 2}-C_{m}^{2}\right), n_{g 1,2}=C_{g 1,2} V_{g 1,2} / e$, and $C_{\Sigma 1,2}=$ $C_{g 1,2}+C_{m}$. In Eq. (11), $n_{1}$ and $n_{2}$ are the numbers of elec-

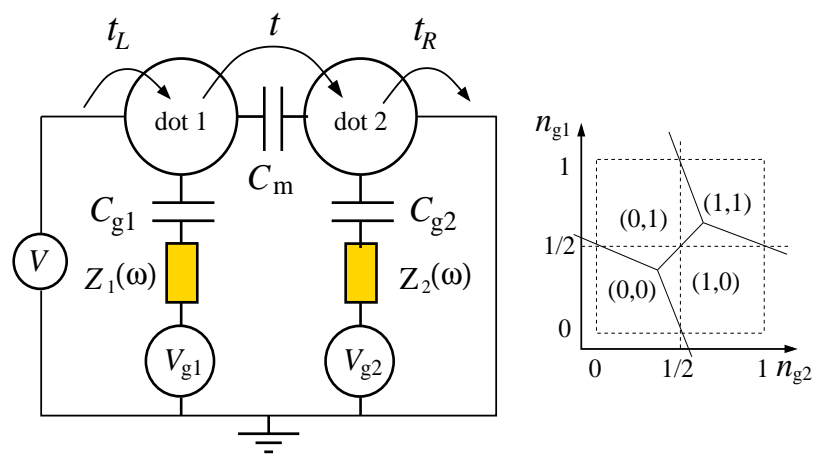

FIG. 1: Left: Schematic setup of a double-QD charge qubit being connected in series to reservoir leads. The two QDs are capacitively coupled to side-gates and fluctuations of the gate voltages are included as a result of the finite resistances $R_{1,2}=Z_{1,2}(\omega=0)$ in the gate leads. Right: Honeycomb pattern of the charging energy for $C_{m} \gg C_{g i}$ where $i=1,2$. Our concern is close to the degeneracy point $n_{g 1}=n_{g 2}=1 / 2$.

trons on each dot with respect to the equilibrium state at $V_{g 1,2}=0$. Our current interest lies in the degeneracy point $n_{g 1}=n_{g 2}=1 / 2$ and in the strong capacitive coupling limit $C_{m} \gg C_{g 1,2}$, so that $E_{01}=E_{10} \ll E_{11}=E_{00}$ with $E_{11}-E_{01}=E_{m} / 2 \simeq E_{c 1} \simeq E_{c 2}$. The low-energy physics can thus be studied within the restricted Hilbert space in which only the $(0,1)$ and $(1,0)$ states are allowed (see Fig. 1). In this case, the double QD effectively reduces to a charge qubit (two-level system) and one can introduce an orbital spin $\hat{T}_{z}$ to describe the qubit, with the orbital spin up and down representing the $(0,1)$ and $(1,0)$ states respectively. It is convenient to introduce the operators $\widehat{P}_{01}$ and $\widehat{P}_{10}$ projecting on the states $(0,1)$ and $(1,0)$ respectively. Through the fluctuation-dissipation theorem, the impedance $Z_{1,2}(\omega)$ introduces gate voltage fluctuations $\delta V_{g 1,2}$. Since $\hat{T}_{z}=\left(\widehat{P}_{01}-\widehat{P}_{10}\right)$, the Hamiltonian describing the charge fluctuations on the charge qubit due to $\delta V_{g 1,2}$ then can be written as $\left(\left\langle\delta V_{g i}(t)\right\rangle=0\right)$

$$
H_{f l}=e\left(\delta V_{g 1}-\delta V_{g 2}\right) \hat{T}_{z} .
$$

We plan to consider the case of Johnson-Nyquist noise 
where the resulting gate voltage fluctuations are embodied by $\left\langle\delta V_{g 1,2} \delta V_{g 1,2}\right\rangle_{\omega}=\hbar R_{1,2} \omega \operatorname{coth}\left(\hbar \omega / 2 k_{B} T\right)$; this can be generally attributed to the presence of ohmic resistances $R_{1,2}$ in the gate leads. Below, we model the resistances $R_{1,2}$ in a microscopic fashion through two long dissipative transmission lines being composed of infinite collections of $L_{i} C_{t i}$ oscillators [Here $i=1,2$ is the dot (and transmission line) index]. For a given gate lead, the resistive external circuit containing the transmission line has been previously introduced in Ref. 9. For the sake of clarity, here we follow Ref. 10 by denoting the charge (fluctuation) operator on the capacitor $C_{g i}$ by $\hat{Q}_{0, i}$ and the ones on the capacitors $C_{t i}$ between the inductances $L_{i}$ by $\hat{Q}_{l, i}$ with $l=1,2, \ldots$, and introducing the corresponding conjugate phase operators $\hat{\phi}_{l, i}$. These transmission lines produce ohmic resistance $R_{i}=\sqrt{L_{i} / C_{t i}}$ and are described by the bosonic Hamiltonian:

$H_{B}=\sum_{i=1,2}\left\{\frac{\hat{Q}_{0, i}^{2}}{2 C_{g i}}+\sum_{l=1}^{+\infty}\left[\frac{\hat{Q}_{l, i}^{2}}{2 C_{t i}}+\frac{\hbar^{2}}{e^{2}} \frac{\left(\hat{\phi}_{l, i}-\hat{\phi}_{l-1, i}\right)^{2}}{2 L_{i}}\right]\right\}$

To diagonalize $H_{B}$ analytically, we assume $C_{t i}=C_{g i}$. Using the transformation 10] $\hat{Q}_{l, i}=\sqrt{2} \int_{0}^{1} d x \cos [(l+$ $1 / 2) \pi x] \hat{Q}_{i}(x)$ and $\hat{\phi}_{l, i}=\sqrt{2} \int_{0}^{1} d x \cos [(l+1 / 2) \pi x] \hat{\phi}_{i}(x)$, with $\left[\hat{\phi}_{j}(x), \hat{Q}_{k}(y)\right]=i e \delta(x-y) \delta_{j k}$, and redefining $\hat{Q}_{i}(x)$ and $\hat{\phi}_{i}(x)$ in terms of boson operators $\hat{a}_{x i}$, we have

$$
H_{B}=\sum_{i=1,2} \int_{0}^{1} d x \hbar \omega_{x i}\left(\hat{a}_{x i}^{+} \hat{a}_{x i}+\frac{1}{2}\right)
$$

where $\omega_{x i}=2 \omega_{c i} \sin (\pi x / 2)$ with $\omega_{c i}=\sqrt{1 / L_{i} C_{t i}}$ being a realistic high-frequency cutoff. The gate voltage fluctuations $\delta V_{g i}=\hat{Q}_{0, i} / C_{g i}$ in Eq. (2) read [10]

$$
\delta V_{g i}=\frac{1}{C_{g i}} \int_{0}^{1} d x \cos \left(\frac{\pi x}{2}\right) \sqrt{\hbar \omega_{x i} C_{t i}}\left(\hat{a}_{x i}+\hat{a}_{x i}^{+}\right) .
$$

It thus becomes clear that Eq. (2) introduces a coupling between the orbital spin and two boson baths.

Now we incorporate electron tunneling between the two QDs. Throughout this paper we imply that a strong in-plane magnetic field has been applied, which allows us to restrict ourselves to spinless electrons. We still have to distinguish between large (metallic) QDs and small QDs. Large QDs are at the micron scale and then yield a very dense energy spectrum. In contrast, a small QD is at the nano scale and the energy level spacing is quite large.

We first consider the case of large QDs. They are in the many-body state $\left|\Phi_{1} \Phi_{2}\right\rangle$ described by the free kinetic Hamiltonian $H_{\mathrm{dot}}^{(0)}=\sum_{i=1,2} \sum_{p_{i}} \epsilon_{p_{i}} d_{i p_{i}}^{+} d_{i p_{i}}$, where $d_{i p_{i}}$ is the annihilation operator for an electron in the state $p_{i}$ on the $i$ th dot. However, the Coulomb blockade effect analyzed above dictates that the tunneling can only take place between the $(0,1)$ and $(1,0)$ states. The key point is that one must use the projection operators $\widehat{P}_{01}$ and
$\widehat{P}_{10}$ to actually project on $\left|\Phi_{1} \Phi_{2}\right\rangle$ with charge number $n_{1}=0, n_{2}=1$ and $n_{1}=1, n_{2}=0$, respectively. The tunneling Hamiltonian then becomes

$$
H_{t}^{\text {(large) }}=t \sum_{p_{1} p_{2}} d_{1 p_{1}}^{+} d_{2 p_{2}} \widehat{P}_{01}+t^{*} \sum_{p_{1} p_{2}} d_{2 p_{2}}^{+} d_{1 p_{1}} \widehat{P}_{10} .
$$

By explicitly introducing an auxiliary charge label $\left|n_{1} n_{2}\right\rangle$ to $\left|\Phi_{1} \Phi_{2}\right\rangle$ the Hamiltonian $H_{t}$ in Eq. (6) then turns into

$$
H_{t}^{(\text {large })}=t \sum_{p_{1} p_{2}} d_{1 p_{1}}^{+} d_{2 p_{2}} \hat{T}_{-}+t^{*} \sum_{p_{1} p_{2}} d_{2 p_{2}}^{+} d_{1 p_{1}} \hat{T}_{+} .
$$

The tunneling matrix element $t$ is assumed not to depend on the momenta $p_{1}$ and $p_{2}$. The $\hat{T}_{+}$operator - acting exclusively on $\left|n_{1} n_{2}\right\rangle$ - ensures that each time an electron travels from dot 1 to dot 2 the charge state is explicitly adjusted from $|10\rangle$ to $|01\rangle=\hat{T}_{+}|10\rangle$. This causes a flip in the orbital spin which is accompanied by particlehole excitations shared between the two dots. We find it appropriate to visualize $H_{t}^{\text {(large) }}$ as a transverse Kondo coupling along the lines of a single-electron box coupled to a reservoir lead [11]. The Kondo energy scale below which the transmission between dots becomes almost perfect reads $T_{K 0}^{(\text {large })}=D_{0} e^{-1 /\left(2|t| N_{0}\right)}$; the ultraviolet energy cutoff is $D_{0} \sim E_{c 1,2}$ and $N_{0}$ is the density of states on the two dots which has been assumed to be symmetric.

The total Hamiltonian for the two large dots in the absence of the leads can be summarized as

$$
H_{\text {dot }}^{(\text {large })}=H_{f l}+H_{B}+H_{t}^{(\text {large })}+H_{\text {dot }}^{(0)} .
$$

This is now clearly mapped onto a spin-boson-fermion model [12]. We find the following renormalization group (RG) equations: $d \lambda_{\perp} / d l=\lambda_{\perp} \lambda_{z}-g \lambda_{\perp} / 2, d \lambda_{z} / d l=$ $\lambda_{\perp}^{2}$, and $d g / d l=-g \lambda_{\perp}^{2}$ with $l=\ln \left(D_{0} / D\right)$ denoting the scaling variable ( $D$ is the energy variable). Here, $\lambda_{\perp}=2|t| N_{0}, \lambda_{z}$ is the induced Ising-like coupling between the orbital spin and the fermion degrees of freedom, and $g$ is an important physical parameter defined as $g=4\left(R_{1}+R_{2}\right) / R_{Q}$ (in the case of $\left.C_{t i}=C_{g i}\right) ; R_{Q}=$ $h / e^{2} \approx 25.8 k \Omega$ is the quantum of resistance. Like in Ref. 9, we rewrite the RG equations in terms of the variables $\lambda_{\perp}=2|t| N_{0}$ and $\tilde{\lambda}_{z}=\lambda_{z}-g / 2$; initially, $\tilde{\lambda}_{z}=-g / 2 \leq 0$. Then, we recognize the well-known Kosterlitz-Thouless (KT) equation flow $d \lambda_{\perp} / d l=\lambda_{\perp} \tilde{\lambda}_{z}$ and $d \tilde{\lambda}_{z} / d l=\lambda_{\perp}^{2}$, which reveals a quantum phase transition at $\tilde{\lambda}_{z}=-\lambda_{\perp}$, i.e., $g=g_{c l}=4|t| N_{0}$ which experimentally corresponds to $R_{1}+R_{2}=|t| N_{0} R_{Q}<R_{Q}$ in the weak tunneling limit $|t| N_{0} \ll 1$. For $g<g_{c l}$, the charge qubit is still in the Kondo realm with the Kondo temperature being renormalized by noise: $T_{K}^{(\text {large })}(g) \approx D_{0} e^{-1 /\left(2|t| N_{0}-g / 2\right)}$; the boson baths have additive effects. For $g>g_{c l}$, the noise effect becomes dominant and eliminates the orbital Kondo effect; the hopping between the two QDs becomes tiny and the current is strongly suppressed (see below).

In contrast to the large QDs, the small QDs are in a truly two-level state at the degeneracy point $n_{g 1}=n_{g 2}=$ 
$1 / 2$ due to the large energy level spacing. Tunneling of electrons between the two QDs still induces an orbital spin flip however there is no particle-hole excitation involved. In consequence, the tunnel Hamiltonian between the two small dots takes the more conventional form [ 6 ]

$$
H_{\text {dot }}^{(\text {small })}=H_{f l}+H_{B}+\left(t \hat{T}_{-}+\text {h.c. }\right) .
$$

Such a model has been already investigated in great detail, including in the context of a small noisy dot embedded in a mesoscopic ring [10]. According to Ref. 10, this can be mapped exactly onto a transverse spin-boson model 13] which in passing has been demonstrated to apply to a wide range of two-level state problems including a superconducting qubit 14 and a quantum dot connected to Bose-Einstein condensate reservoirs [15]. The phase diagram can also be derived from an analogy with the single-impurity (anisotropic) Kondo model [13]. This already ensures some universality in the transport features independently of the precise sizes of the QDs. Here the Kondo energy scale $T_{K}^{(\text {small })}(g)=|t|\left\{|t| / D_{0}\right\}^{g /(1-g)}$ only decays as a power-law with $g$ and that the KT phase transition should arise in the vicinity of $g=g_{c s} \approx 1[10$.

Now, we are in a favorable position to accurately study transport properties through the double-QD charge qubit in the quantum limit of $T=0$. We treat the case of the large dots and the small dots in order.

Transport through large QDs.-We connect the two QDs in series to two leads (source and drain) and add a voltage drop $V$ across the two leads as shown in Fig. 1. In the restricted Hilbert space, at small energy $\mathrm{eV}$, singleelectron sequential tunneling through the dots is exponentially suppressed [16]. The leading order contribution to the tunneling here comes from the cotunneling process:

$$
H_{T}^{(\text {large })}=t^{\prime} \sum_{k_{1} k_{2}} \sum_{p_{1} p_{2}}\left(c_{2 k_{2}}^{\dagger} c_{1 k_{1}} d_{1 p_{1}}^{+} d_{2 p_{2}} \hat{T}_{-}+\text {h.c. }\right),
$$

where $c_{i k_{i}}$ annihilates a spinless electron in the state $k_{i}$ on the left $(i=1)$, right $(i=2)$ lead; $t^{\prime} \simeq t_{L} t_{R} /\left[E_{11}-E_{01}\right]=$ $2 t_{L} t_{R} / E_{m}$ with $t_{L}\left(t_{R}\right)$ being the tunneling matrix element between the left lead and dot 1 (the right lead and dot 2). Since $H_{T}^{(\text {large) }}$ involves four fermion operators, this is irrelevant in the RG spirit and can always be treated perturbatively.

To compute the tunneling current, it is convenient to absorb the coupling to the quantum noise into the tunneling term through the canonical transformation $\mathcal{U}=$ $\exp \left\{i \widehat{T}_{z}\left(\hat{\phi}_{0,1}-\hat{\phi}_{0,2}\right)\right\}$, where $\hat{\phi}_{0, i}=\frac{e}{\hbar} \int^{t} d t^{\prime} \delta V_{g i}\left(t^{\prime}\right) d t^{\prime}$. The Hamiltonian (10) then turns into:

$$
\begin{aligned}
& \widetilde{H}_{T}^{\text {(large })}=t^{\prime} \widehat{A}+\text { h.c. } \\
& \widehat{A}=\sum_{k_{1} k_{2}} \sum_{p_{1} p_{2}} c_{2 k_{2}}^{\dagger} c_{1 k_{1}} d_{1 p_{1}}^{+} d_{2 p_{2}} \hat{T}_{-} e^{i\left(\hat{\phi}_{0,1}-\hat{\phi}_{0,2}\right)} .
\end{aligned}
$$

By using linear response theory, the tunneling current then can be expressed as 17.

$$
I_{L}=-2 e\left|t^{\prime}\right|^{2} \operatorname{Im}\left\langle\widehat{A} \cdot \widehat{A}^{+}\right\rangle_{\mathrm{ret}}(-e V),
$$

where $\langle\cdots\rangle_{\text {ret }}$ means the retarded correlation function computed with respect to the states of $H_{\mathrm{dot}}^{(\text {large })}$. It is important to note that the Hamiltonian in (10) alone does not drive a current because it conserves the total number of electrons in the left lead and in the left dot [6]. It is therefore necessary to have the tunneling term between the two QDs, $H_{t}^{\text {(large) }}$, in order to obtain a nonvanishing tunneling current. We immediately infer that in the boson phase $g>g_{c l}$, the current is negligible as a consequence of the suppressed tunneling between the two dots due to the fluctuating electromagnetic fields.

Now we focus on the Kondo regime $g<g_{c l}$. As discussed before, the Kondo coupling $\lambda_{\perp}(l)$ in $H_{t}^{\text {(large) }}$ flows to the strong coupling limit resulting in a perfect transmission between the two dots. To the leading order, we then expect $\left\langle\sum_{p_{1} p_{2}} d_{1 p_{1}}^{+} d_{2 p_{2}} \hat{T}_{-} \cdot \sum_{p_{3} p_{4}} d_{2 p_{4}}^{+} d_{1 p_{3}} \hat{T}_{+}\right\rangle(\omega) \sim$ $\delta(\omega)$ as a result of the formation of an Abrikosov-Suhl resonance at zero frequency $(\omega=0)$ which is the signature of a Kondo ground state, and thus

$$
\begin{aligned}
& I_{L}=2 \pi e\left|t^{\prime}\right|^{2} N_{L} N_{R} \int_{-\infty}^{\infty} d \xi \int_{0}^{\omega_{c}} d \omega_{1} \int_{0}^{\omega_{c}} d \omega_{2} f(\xi) \\
& \times\left[1-f\left(\xi+e V+\hbar \omega_{1}+\hbar \omega_{2}\right)\right] \mathcal{P}_{1}\left(\omega_{1}\right) \mathcal{P}_{2}\left(\omega_{2}\right) .
\end{aligned}
$$

Here $f(\xi)$ is the Fermi function (At low $e V$, we can restrict ourselves to equilibrium states [18, 19]), $N_{L}$ and $N_{R}$ are the density of states in the left and right leads, and we introduce the following Fourier transform

$$
\begin{aligned}
\mathcal{P}_{i}(\omega) & =\int_{0}^{\infty} d t e^{i \omega t}\left\langle e^{i \hat{\phi}_{0, i}(t)} e^{-i \hat{\phi}_{0, i}(0)}\right\rangle \\
& \simeq\left[\exp \left(-\alpha_{i} \gamma_{0}\right) / \Gamma\left(\alpha_{i}\right)\right] \omega^{\alpha_{i}-1} / \omega_{c}^{\alpha_{i}},
\end{aligned}
$$

for $\omega \ll \omega_{c}$, where $\alpha_{i}=2 R_{i} / R_{Q}$ (so $\left.\alpha_{1}+\alpha_{2}=g / 2\right), \gamma_{0} \simeq$ 0.5772 is Euler's constant, and $\Gamma(x)$ the Gamma function. For convenience, we have assumed that $\omega_{c_{1}}=\omega_{c_{2}}=\omega_{c}$. The quantity $\mathcal{P}_{i}\left(\omega_{i}\right)$ has a well-defined mesoscopic interpretation [16, 18, 19]: this represents the probability that an electron which tunnels onto the dot $i$ also creates a charge excitation with an energy $\hbar \omega_{i}$ in the dissipative environment $i$. From Eq. (14) it becomes apparent that the cotunneling of electrons across the double dot must be mediated through excitations in the two environmental bosonic modes; this will be referred to as "dissipative cotunneling". Inserting Eq. (15) into Eq. (14) yields

$$
I_{L}=c_{L}\left[\exp \left(-g \gamma_{0} / 2\right) / \Gamma(2+g / 2)\right]\left(e V / \hbar \omega_{c}\right)^{1+g / 2}
$$

at $T=0$, where $c_{L}=\left|t^{\prime}\right|^{2} N_{L} N_{R} \hbar \omega_{c} / e R_{Q}$. Again, we see that the two noises have additive effects. In Fig. 2, we plot $I_{L}$ normalized to $c_{L}$ as a function of $e V / \hbar \omega_{c}$ at various $g$. We discern that in the Kondo regime the $I-V$ characteristic curves of the double-QD charge qubit can be viewed as an interesting generalization of the ones of the noisy tunnel junction [18, 19] and QD [19]. The decreasing current with growing $g$ at small voltage can be 


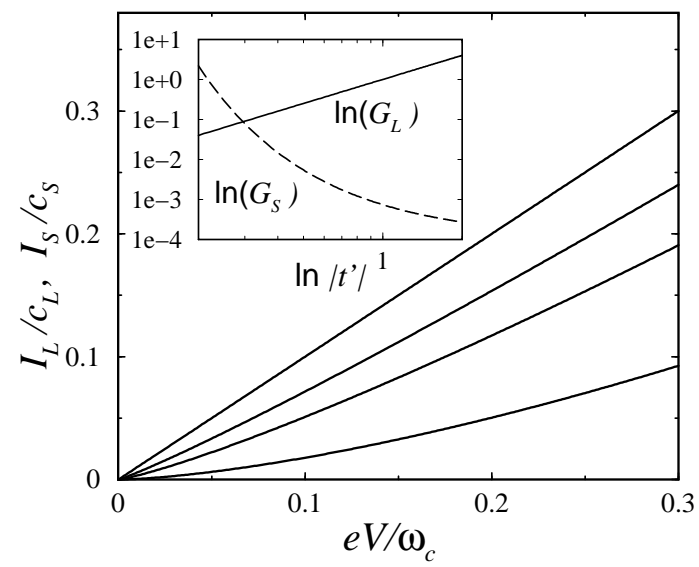

FIG. 2: Normalized currents $I_{L} / c_{L}$ and $I_{S} / c_{S}$ as a function of the dimensionless bias voltage $\mathrm{eV} / \omega_{c}(\hbar=1)$ in the Kondo regime. Curves from top to bottom correspond to $g / 2=\alpha_{1}+\alpha_{2}=0,0.1,0.2,0.5$. The inset is a log-log plot of the conductance for large dots (solid line) and for small dots (dashed line) versus $\left|t^{\prime}\right|$ in the Kondo regime in the specific situation where $g=0$ and $T_{K L} \gg T_{K}^{(\text {small })}(g)\left(|t| / D_{0}=0.0001\right)$.

associated with the more and more suppressed noise spectrum at small $\omega$. In the absence of the noises, the conductance obeys $G_{L}=\left|t^{\prime}\right|^{2} N_{L} N_{R} / R_{Q}$ similar to a large $\mathrm{QD}$ at the Coulomb blockade peaks [16].

Transport through small QDs.-When the two small QDs are connected in series to the leads, the cotunneling Hamiltonian becomes $H_{T}^{\text {(small) }}=t^{\prime} \sum_{k_{1} k_{2}}\left(c_{2 k_{2}}^{\dagger} c_{1 k_{1}} \hat{T}_{-}+\right.$ h.c.). Here $H_{T}^{\text {(small) }}$ can also generate a Kondo effect at the energy scale $T_{K L}=D_{0} e^{-1 /\left(2\left|t^{\prime}\right| N_{l}\right)}$ (we have assumed $N_{L}=N_{R}=N_{l}$ ). For quite reasonable $t$, in principle, we should have $T_{K}^{(\text {small })}(g) \gg T_{K L}$ which guarantees the validity of our perturbative treatment of $H_{T}^{\text {(small) }}$, i.e., Eq. (16) found previously for the large dots is then applicable. Eventually, by converging to the extreme limit of very small $t, H_{T}^{\text {(small) }}$ could dominate the orbital Kondo physics and then the leading order term in the current becomes quadratic in $t[\underline{6}]$. The current at $T=0$ reads

$$
I_{S}=c_{S}\left[\exp \left(-g \gamma_{0} / 2\right) / \Gamma(2+g / 2)\right]\left(e V / \hbar \omega_{c}\right)^{1+g / 2},
$$

where $c_{S}=\left|t / T_{K L}\right|^{2} \hbar \omega_{c} / e R_{Q}$. For $g=0$, we recover the conductance $G_{S}=\left|t / T_{K L}\right|^{2} / R_{Q}$ [6]. Both $I_{L}$ in Eq. (16) and $I_{S}$ in Eq. (17) have the same power law, albeit due to different orbital Kondo physics. A comparison between $G_{L}$ and $G_{S}$ versus $\left|t^{\prime}\right|$ has been performed in Fig. 2 (inset). Note that orbital Kondo physics differs from the conventional spin Kondo effect in which the perfect transmission usually leads to the conductance quantum $2 / R_{Q}$ (the factor 2 is attributed to the two spin channels).

In conclusion, we have carefully investigated the effect of gate voltage fluctuations on transport through a
double-QD charge qubit. We have obtained striking I-V characteristics as a result of the prolific combination between orbital Kondo entanglement and dissipative cotunneling (the transfer of electrons through the structure has to be conducted by bosonic excitations in the two electromagnetic environments). This generalizes previous noise studies on the single tunnel junction and QD. When the coupling to the dissipative environments becomes large enough the orbital Kondo effect is suppressed and the orbital spin acts like a free moment which completely depletes the current (at low $V$ ). A similar analysis can be performed with superconducting leads and dots.

We thank L. Borda and G. Zaránd for discussions. This work was funded by CIAR, FQRNT and NSERC.

[1] See, e.g., L. Kouwenhoven and L. Glazman, Phys. World 14, 33, (2001).

[2] See, e.g., H. von Löhneysen, T. Pietrus, G. Portisch, H. G. Schlager, A. Schröder, M. Sieck, and T. Trappmann, Phys. Rev. Lett. 72, 3262 (1994).

[3] See, e.g., Q. Si, S. Rabello, K. Ingersent, and J. L. Smith, Nature (London) 413, 804 (2001).

[4] N. Andrei, G. Zimányi and G. Schön, Phys. Rev. B 60, R5125 (1999).

[5] Y. Pashkin, T. Yamamoto, O. Astafiev, Y. Nakamura, D. V. Averin, and J. S. Tsai, Nature 421, 823 (2003).

[6] L. Borda, G. Zaránd, W. Hofstetter, B. I. Halperin, and J. von Delft, Phys. Rev. Lett. 90, 026602 (2003).

[7] F. R. Waugh, M. J. Berry, D. J. Mar, R. M. Westervelt, K. L. Kampman, and A. C. Gossard, Phys. Rev. Lett. 75, 705 (1995); U. Wilhelm and J. Weis, Physica E 6, 668 (2000).

[8] N. Mason, M. J. Biercuk, and C. M. Marcus, Science 303, 655 (2004).

[9] K. Le Hur, Phys. Rev. Lett. 92, 196804 (2004).

[10] P. Cedraschi and M. Büttiker, Annals of Physics 289, 1 (2001).

[11] K. A. Matveev, Zh. Eksp. Teor. Fiz. 99, 1598 (1991) [Sov. Phys. JETP 72, 892, (1991)].

[12] L. Zhu and Q. Si, Phys. Rev. B 66, 024426 (2002); G. Zaránd and E. Demler, ibid. 66, 024427 (2002).

[13] A. J. Leggett, S. Chakravarty, A. T. Dorsey, M. P. Fisher, A. Garg, and W. Zwerger, Rev. Mod. Phys. 59, 1 (1987).

[14] Y. Makhlin, G. Schön, and A. Shnirman, Rev. Mod. Phys. 73, 357 (2001);

[15] A. Recati, P. O. Fedichev, W. Zwerger, J. von Delft, and P. Zoller, cond-mat/0404533

[16] G. Schön in Quantum Transport and Dissipation, edited by T. Dittrich, P. Hänggi, G.-L. Ingold, B. Kramer, G. Schön, and W. Zwerger (WILEY-VCH, Weinheim, 1998), Chap. 3, pp. 157-159 and pp. 163-172.

[17] G. D. Mahan, Many-Particle Physics, (Plenum press, New York and London, 1993).

[18] M. H. Devoret, D. Esteve, H. Grabert, G.-L. Ingold, H. Pothier and C. Urbina, Phys. Rev. Lett. 64, 1824 (1990).

[19] Yu. V. Nazarov and G.-L. Ingold in Single Charge Tunneling, edited by H. Grabert and M. H. Devoret (Plenum Press, New York, 1992), Chap. 2, pp. 21-107. 